\title{
Observations on interphase characterisation in polymer composites by nano-scale indentation and the use of AFM cantilever torsion to identify measurement artefacts
}

\author{
T. J. Young ${ }^{1 \star}$, M. Monclus ${ }^{1}$, W. R. Broughton ${ }^{1}$, S. L. Ogin ${ }^{2}$ and P. A. Smith ${ }^{2}$ \\ ${ }^{1}$ National Physical Laboratory, Hampton Road, Teddington, TW11 0LW, UK \\ ${ }^{2}$ The University of Surrey, Guildford, GU2 7XH, UK. * Tim.young@npl.co.uk
}

\section{SUMMARY}

The results presented in this paper explore the extent to which indentation in the interfacial region of polymer composites can be used to identify, unambiguously, an interphase of distinct elastic properties. Supporting evidence is presented in the form of AFM-indentation in the interfacial region of two polymer composites, a glass fibre-reinforced vinylester and a glass flake-reinforced polypropylene, where a transition region of measurement artefact is revealed as opposed to a physically distinct interphase. The conclusions from these tests are augmented by further work on a glass fibre-reinforced phenolic using a new in-situ method of identifying measurement artefacts.

Keywords: Mechanical characterisation, indentation, interfaces, Atomic Force Microscopy, Force Volume, Elastic Modulus, Composite, Interface, Lateral Force Microscopy

\section{INTRODUCTION}

A commonly held perception is that results of indentation studies, typically instrumented nano-indentation testing (IIT) [1], across the interface of polymer composites reveal an interphase region of mechanically different properties to the matrix or reinforcement [2-6]. High resolution characterisation of the interfacial region using an atomic force microscope (AFM) suggested a distinct stiffness difference between the reinforcement, interphase and matrix with a transition region between each phase [7]. Griswold et al [8], suggest that the interphase may be associated with the introduction of coupling agents on the fibre surface to promote adhesion between the reinforcement and the matrix. In their work, results from a combination of AFM imaging and nanoindentation measurements were used to suggest that the interphase thickness correlated with the concentration of applied coupling agent. They recognised, however, that at small interphase thicknesses (of the order of $210 \mathrm{~nm}$ ) nanoindentation measurements may be affected by the proximity of the fibre. It appears then, that transition regions may not be representative of the material properties and it has been suggested by other researches, that observations of such a transition at a fibre-matrix interface may be the consequence of a locally constrained matrix region in the vicinity of the reinforcement $[9,10]$. This suggestion is consistent with Finite Element Analysis work, which indicates that even a perfect interface would lead to an observed interphase region of apparently varying elastic properties [6]. It has been suggested further by Gao et al [11] and Munz et al [12] that such a zone of interaction encompasses a distance of about 2-3 times the size of the indenter contact area away from the interface. Such studies have indicated 
that point measurements across an interface may reveal measurement artefacts rather than variation in mechanical properties.

Currently, there is no established in-situ method of separating transition effects associated with the measurement technique from any mechanical property variation that might be associated with an interphase. The aim of this paper is to report on quantitative AFM indentation experiments and identify whether true interphase regions can be detected within glass fibre- and flake-reinforced polymer systems. Results will be presented relating to AFM indentation experiments across the interface of two polymer composites (glass fibrereinforced vinylester and glass flake-reinforced polypropylene) in which measurement artefacts are identified as opposed to true interphase properties. These results are complemented by data obtained on a third composite (glass fibre-reinforced phenolic) for which a novel in-situ method for identifying measurement artefacts is developed.

\section{EXPERIMENTAL METHODS}

\section{Introduction}

In this section the instrumentation used for indentation by AFM is described along with the details of a MATLAB data analysis algorithm used to analyse the indentation data.

Supporting experimental work using an Instrumented Nano-Indenter (IIT) is also outlined. Details of the experimental procedures and test materials are then provided.

\section{Instrumentation}

A Park XE-100 (Parks Systems, Suwon, KR) was used for the AFM indentation experiments with a sharp diamond tip with a $20.5 \mathrm{~nm}$ radius mounted on a $200 \mathrm{~N} \mathrm{~m}^{-1}$ (calibrated by Microstar [13]) sapphire cantilever, manufactured by MicroStar (Huntsville, USA). A Zeiss Supra Scanning Electron Microscope (SEM) (Carl Zeiss SMT AG, Germany) was used to measure the geometry of the AFM cantilever to confirm, using Euler-Bernoulli beam theory $[14,15]$, the Microstar-calibrated spring constant. The dimensions of the AFM tip and cantilever are given in Table 1; the calculated stiffness of the beam is $214 \mathrm{~N} \mathrm{~m}^{-1}$, which is in reasonable agreement with the value from the supplier of $200 \mathrm{~N} \mathrm{~m}^{-1}$.

\section{INSERT TABLE 1}

During indentation, the torsion of the cantilever was recorded using a LabVIEW (National Instruments, Texas, USA) interface. Figure 1 shows a schematic of the detection system used to measure movement of the cantilever during indentation. The voltage difference between the photosensitive diodes $A$ and $B$ relates principally to bending of the cantilever (normal force at the tip) while the difference between $C$ and $D$ relates principally to the torsion of the cantilever (lateral force at the tip).

\section{INSERT FIGURE 1}

A data analysis algorithm was written in MATLAB (Mathworks, MA) in order to deal with the large number of indentations required for calibrating the AFM system and mapping a surface. Each measured force-displacement curve required the point of contact to be determined in order to separate the approach of the tip towards the surface from the indentation of the sample. The measured parameters are the A-B voltage and the distance the cantilever has moved towards the surface. Raw voltage-distance curves (Figure 2a) are reversed about the 
point of contact (Figure $2 \mathrm{~b}$ ) and then cropped to retain the indentation portion of the voltagedistance curve (Figure 2c). The point of contact was taken to correspond to the first of three data points measured above a set threshold. This threshold was set, typically, to a value of $0.1 \%$ above the measured voltage during the approach. In this way, the effect of noise triggering a false point of contact in the MATLAB data analysis algorithm was avoided.

\section{INSERT FIGURE 2}

IIT experiments were performed using an MTS Nano Indenter instrument (MTS, Oak Ridge, Tennessee, USA) with a Berkovich indenter and a maximum indentation force of $1 \mathrm{mN}$. The indenter instrument was used in continuous stiffness mode, suited for materials exhibiting viscoelastic behaviour [1]. For these experiments, the oscillation frequency was set to $40 \mathrm{~Hz}$ with a force amplitude of $3 \mu \mathrm{N}$. A set of reference samples (with modulus values certified by IIT) was used to calibrate the AFM; the reference samples included sapphire, fused silica, a polymeric photo-stress coating (denoted PS-1), ABS resin and PMMA.

\section{Experimental Procedure}

The experimental procedure was carried out as follows. Prior to indentation the AFM tip radius was measured using the SEM and the cantilever spring constant quoted by the supplier was verified. The system was calibrated by indentation on sapphire, to determine the frame compliance [16], and on reference samples, to determine the effective tip radius $[16,17]$. The composite samples were indented appropriately and the elastic modulus was calculated using a Hertzian model [19]. The same reference samples were indented again to check for parameter changes and the tip was examined using the SEM for changes as a result of wear.

From the results obtained, the reduced elastic modulus was calculated using a Hertzian model (equations 1 and 2 below) with the effective tip radius, $R$, established using the reference samples [17].

$$
\begin{aligned}
& E_{r}=\frac{3 \alpha}{4 \sqrt{R}} \\
& \alpha=\frac{F}{h^{n}}
\end{aligned}
$$

In equation 2, $F$ is the force at indentation depth, $h$, and both $\alpha$ and $n$ are constants $(n=1.5$ for an ideal Hertzian contact).

The reduced elastic modulus, $E_{r}$, is related to the sample elastic modulus, $E_{s}$, by

$$
\frac{1}{E_{r}}=\frac{\left(1-v_{s}^{2}\right)}{E_{s}}+\frac{\left(1-v_{I}^{2}\right)}{E_{I}}
$$

where $E_{l}$ is the indenter elastic modulus, $v_{I}$ is the Poisson's ratio of the indenter and $v_{s}$ is the Poisson's ratio of the sample [16]. 
Hertzian contact mechanics assumes that the whole contact is spherical and the power law exponent, $n$, from $F=\alpha h^{n}$ is equal to 1.5 , whereas the experimentally obtained value of $n$ was between 1.3 and 1.5. This is consistent with earlier work [18] and may be influenced by a number of factors, including an imperfect spherical geometry, rotation of the AFM tip or an indentation where a small portion of the contact is on the cone (when making partial contact with the interface).

\section{Materials}

A variety of materials with different interfacial treatments have been used in these experiments (as indicated below, each of these systems has the potential to exhibit an interphase region). The surface preparation method used for the samples was a modified version of the mechanical polishing method employed by Khanna et al [20] with additional ultrasonic bath treatment. The materials were as follows:

- Glass flake-reinforced polypropylene with $0.28 \mathrm{wt} \%$ aminosilane applied to the glass flakes prior to processing (supplied by NGF Europe). The glass flakes were of random geometry and dispersion. In previous work [6], indentations across the interface of other polypropylene composites suggested the presence of sub-1 $\mu \mathrm{m}$ transitions of distinct elastic moduli.

- Two continuous unidirectional glass fibre-reinforced vinylester pultruded rods, one sample with "poor" interfacial bonding and the other with "good" interfacial bonding (supplied by Exel Composites, UK). The "poor" system showed inferior matrix dominated mechanical properties to the system with "good" interfacial properties. AFM results of the "poor" system are reported here.

- A phenolic resin, Resinox CL1880 mixed with 7 wt\% H1196 hardener reinforced with 450 $\mathrm{g} / \mathrm{m}^{2}$ E-type glass fibres of $20 \mu \mathrm{m}$ diameter coated with an aminosilane coupling agent (supplied by Dr Alma Hodzic, University of Sheffield, UK). In previous work [3] the authors identified a $6 \mu \mathrm{m}$ interphase region between the glass fibre and phenolic-resin after water aging for 10 weeks at $23^{\circ} \mathrm{C}$ and the material in the present study was aged in the same way.

\section{RESULTS}

\section{Introduction}

This section is separated into three parts, with results presented first from AFM indentation across the interface of the glass flake-reinforced polypropylene and then the glass fibrereinforced vinylester samples. Finally, the application of a new method of identifying measurement artefacts, associated with restriction of the indentation by the reinforcement, is demonstrated with the aid of results from the glass fibre-reinforced phenolic resin system.

\section{Glass flake-reinforced polypropylene}

AFM indentations on the glass flake-reinforced polypropylene specimen were performed using the MicroStar diamond indenter. The radius of the indenter was measured by SEM to be about $25 \mathrm{~nm}$ (a more precise measurement was not possible because of the effect of charging of the tip). Based on a reference set of AFM experiments using fused silica with a modulus of $69 \mathrm{GPa}$ (measured using IIT), the effective radius of the tip was calculated to be $20.5 \mathrm{~nm}$, following the reference sample method [16, 17]. Figure 3 shows indentation results 
on the same fused silica reference sample after completion of the glass flake-reinforced polypropylene experiments. The measured modulus now was $71 \mathrm{GPa}$, suggesting that the tip parameters had not changed to any significant degree during the AFM indentation experiments.

\section{INSERT FIGURE 3}

Figure 4 shows data for modulus as a function of distance for an array of indentations over an area of $9 \mu \mathrm{m} \times 2.5 \mu \mathrm{m}$ spanning the interface. The indentation separation was $70 \mathrm{~nm}$ and the maximum indentation width and depth (in the polypropylene) were $54 \mathrm{~nm}$ and $45 \mathrm{~nm}$, respectively. On inspection of the surface subsequently, there was no observable residual imprint and consequently it was not possible to identify the location of any of the indentations. From these results, we note first that the technique gives reasonable values of modulus for the flake and matrix regions. At the interface a very short transition region of 1-2 indentations is apparent. It is not possible to identify whether the transition reflects a change in material properties (associated with an interphase) or is a consequence of a restriction of the indentation process. However, the transition width is less than three times the geometric width of the indenter, which is within the region where interaction with the fibre is likely [10, $11]$.

Overall, the results, presented in Figure 4 show no clear interphase region, although the presence of an interphase with a very small thickness (no more than $150 \mathrm{~nm}$ ) cannot be discounted.

\section{INSERT FIGURE 4}

Figure 5a shows the reduced elastic modulus results of the IIT measurements on the glass flake-reinforced polypropylene sample, where the residual imprint (as shown in Figure 5b) enables the location of the 15 indentations with respect to the reinforcement to be identified. Indentations 7, 8,11 and 14 overlapped the matrix/fibre interface and the associated modulus results were between those measured for the centre of the glass flake (around $60 \mathrm{GPa}$, based on indentations 10 and 12) and the polypropylene (2.7 GPa to 4.4 GPa, based on indentations 2 to 6 ). It is noted that the elastic modulus measured for the matrix is somewhat higher than expected for bulk polypropylene. This could be a consequence of the high local strain rates around the indenter; the polypropylene might also be expected to show a higher modulus for small-strain measurements. Figure 5 shows also that modulus measurements near the edge of the flake (e.g. $42 \mathrm{GPa}$ for indentation 15) are lower than those in the centre of the flake. This may be due to a slight rotation of the flake under loading.

\section{INSERT FIGURE 5}

Modulus measurements near the flake edge and matrix edge (e.g. indentations 8 and 15) give values that are between those measured on the flake and in the matrix. As such, these values cannot be taken to be representative of a distinct interphase region.

\section{Glass fibre-reinforced vinylester}

In a second set of experiments, the continuous unidirectional glass fibre-reinforced vinylester pultruded rod with the "poor" interface was evaluated. Preliminary transmission electron microscopy studies had indicated the possible presence of an interphase region in this 
system [21] and transverse fracture surfaces show the presence of the coupling agent used by the manufacturer (see Figure 6). High resolution AFM results are presented here for the poorly bonded system.

\section{INSERT FIGURE 6}

A high-resolution array of AFM indentations in the interface region of this material was performed (see Figure 7). Figure 7a shows elastic modulus as a function of distance for an array of AFM indentations over an area of $5 \mu \mathrm{m} \times 1.95 \mu \mathrm{m}$ spanning the interface. Regions corresponding to the vinylester matrix and glass reinforcement are indicated in Figure $7 \mathrm{a}$, together with a transition region of intermediate elastic modulus. Figure $7 \mathrm{~b}$ shows three lines of indentations (from the array in Figure 7a) that suggest a transition region of about $200 \mathrm{~nm}$ between the reinforcement and matrix. Figure 7 also shows a region of significantly lower elastic modulus (compared with the vinylester). Overall, the transition region shown in Figure 7 is approximately four times the size of the indentation width, perhaps suggesting that the change in material property may only be partially attributed to measurement artefact $[10,11]$. Two possible contributions to the observed transition region are considered below.

\section{INSERT FIGURE 7}

Firstly, there is the possibility of an interphase of varying elastic modulus within the transition region. This is supported by the evidence of an interphase or coupling agent on the surface of the glass fibre (Figure 6). A difficulty with this interpretation is that there were no residual imprints that could be used to identify which indentations could have been restricted by the reinforcement. For this reason, it is not conclusive that the measurements in the transition region are purely a product of interphase material. Secondly, the effective modulus may be influenced by the presence of interfacial failure. Both optical and scanning electron microscopy of the surface suggested that debonds were present.

It is clear from the above that the interpretation of AFM data would be facilitated by the development of a technique that can identify measurement artefacts. Such a technique is outlined in the next section and is then demonstrated with reference to measurements across the interface of a glass fibre-reinforced phenolic specimen.

\section{Detection of artefacts}

A technique for detecting measurement artefacts by monitoring the restriction of the indentation due to the reinforcement has been developed. When an indentation takes place on an inhomogeneous surface, such as a glass-polymer interface, there will be a lateral force at the AFM tip and an associated torsion of the cantilever (see Figure 8). It is suggested that where there is a significant lateral force present, changes in the elastic modulus within the transition region cannot be differentiated from possible constraint effects.

Figure 9 shows a height profile of the surface of the glass fibre-reinforced phenolic system obtained using non-contact AFM imaging (scanning the AFM tip over the surface without making direct contact to build a topographic image). This was used to locate a suitable area for investigation (i.e. one that was free of cracks and debonds); similar images were taken after indentation to confirm that there was no residual deformation (for these experiments, the AFM tip was $85 \mathrm{~nm} \pm 10 \mathrm{~nm}$ ). 


\section{INSERT FIGURE 8}

\section{INSERT FIGURE 9}

Figure 10 shows voltage-distance curves for the bulk phenolic resin, the glass reinforcement and at a location close to the interface. The voltages were measured by the photosensitive diode (see Figure 1) and relate to the normal forces (A-B) and torsional forces (C-D).

\section{INSERT FIGURE 10}

The torsion curves for the (bulk) phenolic and glass fibre (see Figure 10), have a small gradient suggesting that the tip is not located on the centre line of the cantilever, which results in a rotation during indentation. This small gradient is in contrast to the much larger gradient of the torsion curve (see Figure 10) when the tip is located in the transition region (see Figure 8) corresponding to a larger tip rotation.

The reduced elastic moduli of the phenolic and the glass fibre were derived from the normal force (A-B) indentation curves to be $4.4 \pm 0.5 \mathrm{GPa}$ and $76 \pm 12 \mathrm{GPa}$, respectively (the uncertainties are standard deviations from the whole data set). The measured modulus of the phenolic is more reasonable than the modulus found with a smaller tip radius for the polypropylene. The improved accuracy may indicate that Hertzian mechanics is most appropriate for a larger tip radius (the tip radius was $85 \mathrm{~nm}$ when measuring the phenolic compared with $20.5 \mathrm{~nm}$ when measuring the polypropylene).

With regard to the interphase in this system, it was expected that for the glass fibrereinforced phenolic sample there would be at least a $6 \mu \mathrm{m}$ interphase after water aging [3]. Two lines of indentations $25 \mu \mathrm{m}$ and $10 \mu \mathrm{m}$ across the interface are shown in Figure 11, but the results show no detectable interphase. In each set of results, a single measurement with an elastic modulus higher than that of the matrix (6.1 GPa and 7.2 GPa) was noted, although in each case a significant torsion gradient was measured indicative of a lateral force and interaction with the reinforcement.

\section{INSERT FIGURE 11}

\section{INSERT FIGURE 12}

In order to establish whether the torsion identified in these two cases was the result of a contact with the reinforcement, a second set of measurements (lines of $5 \mu \mathrm{m}$ and $2.5 \mu \mathrm{m}$ ), with a tighter packing of indentations, was performed over the same interface. All of the data from the four sets of measurements are shown in Figure 12 and the data are consistent with a transition region of intermediate reduced elastic modulus, of width $150 \mathrm{~nm}$; however, for each of the measurements within this region, a significant torsion gradient was recorded confirming that the measured changes in the reduced elastic moduli are indistinguishable from measurement artefacts. This interpretation is consistent with the suggestions of Gao et al [11] and Munz et al [12] that any transition region within two to three times the indentation diameter is associated with restriction of the indentation (the maximum indentation diameter here was $120 \mathrm{~nm}$ ). 


\section{CONCLUDING REMARKS}

Transition regions of intermediate elastic modulus were observed for both the glass flakereinforced polypropylene and the glass fibre-reinforced vinylester samples. In each case, the elastic modulus variation cannot be separated from possible measurement artefacts due to restriction of the indentation as a result of the proximity of the reinforcement or the presence of a debond.

A technique for identifying possible measurement artefacts has been developed and demonstrated on a glass fibre-reinforced phenolic composite. The technique involves monitoring the changes in torsional force on the indenter due to inhomogeneous indentation. The results on the glass fibre-reinforced phenolic system suggested a transition region of $150 \mathrm{~nm}$ within which there was a significant torsional force on the indenter. This transition region of varying elastic modulus was less than two to three times the size of the contact area indicating, in agreement with previous work, interaction between the AFM tip and the reinforcement. The technique of monitoring torsional forces on the AFM tip is therefore proposed as a method of identifying elastic modulus measurement artefacts in the region of a fibre-matrix interface.

\section{ACKNOWLEDGEMENTS}

This work was supported by the National Physical Laboratory as part of a Department for Business, Innovation and Skills (BIS) funded project and an Engineering and Physical Sciences Research Council (EPSRC) funded Engineering Doctorate studentship. The authors would like to thank Dr John Nunn of the National Physical Laboratory for his guidance and help with computer programming.

\section{REFERENCES}

[1] W. Oliver and G. Pharr, "Measurement of hardness and elastic modulus by instrumented indentation: Advances in understanding and refinements to methodology," Journal of Materials Research, vol. 19, no. 1, pp. 3-20, 2004.

[2] A. Hodzic, Z. H. Stachurski, and J. K. Kim, "Nano-indentation of polymer-glass interfaces Part I. Experimental and mechanical analysis," Polymer, vol. 41, no. 18, pp. 6895-6905, 2000.

[3] A. Hodzic, J. K. Kim, and Z. H. Stachurski, "Nano-indentation and nano-scratch of polymer/glass interfaces. II: model of interphases in water aged composite materials," Polymer, vol. 42, no. 13, pp. 5701-5710, 2001.

[4] A. Hodzic, S. Kalyanasundaram, J. K. Kim, A. E. Lowe, and Z. H. Stachurski, "Application of nano-indentation, nano-scratch and single fibre tests in investigation of interphases in composite materials," Micron, vol. 32, no. 8, pp. 765-775, 2001.

[5] J. Kim, "Nanoscale characterisation of interphase in silane treated glass fibre composites," Composites Part A: Applied Science and Manufacturing, vol. 32, no. 5, pp. 607-618, 2001.

[6] S. H. Lee, S. Wang, G. M. Pharr, and H. Xu, "Evaluation of interphase properties in a cellulose fiber-reinforced polypropylene composite by nanoindentation and finite element analysis," Composites Part A: Applied Science and Manufacturing, vol. 38, no. 6, pp. 1517-1524, 2007. 
[7] M. R. VanLandingham, R. R. Dagastine, R. F. Eduljee, R. L. McCullough, and J. W. GillespieJr, "Characterization of nanoscale property variations in polymer composite systems: 1. Experimental results," Composites Part A: Applied Science and Manufacturing, vol. 30, no. 1, pp. 75-83, 1999.

[8] C. Griswold, W.M. Cross, L. Kierengtroen and J.J. Keller, "Interphase variation in silanetreated glass-fiber-reinforced epoxy composites", Journal of Adhesion Science and Technology, vol. 19, no 3-5, pp 279-290, 2005.

[9] A. Matzenmiller and S. Gerlach, "Parameter identification of elastic interphase properties in fiber composites," Composites Part B: Engineering, vol. 37, no. 2, pp. 117126, 2006.

[10] P. P. Gohil and A. Shaikh, "Analytical Investigation and Comparative Assessment of Interphase Influence on Elastic Behavior of Fiber Reinforced Composites," Journal of Reinforced Plastics and Composites, vol. 29, no. 5, pp. 685-699, 2009.

[11] S. L. Gao and E. Mäder, "Characterisation of interphase nanoscale property variations in glass fibre reinforced polypropylene and epoxy resin composites," Composites Part A: Applied Science and Manufacturing, vol. 33, no. 4, pp. 559-576, 2002.

[12] W. Possart and European Conference on Adhesion, Adhesion : current research and applications. Weinheim ;[Chichester: Wiley-VCH ;;John Wiley distributor], 2005.

[13] B. Mesa and S. Magonov, "Novel diamond/sapphire probes for scanning probe microscopy applications," Journal of Physics: Conference Series, vol. 61, pp. 770-774, 2007.

[14] W. Young, Roark's formulas for stress and strain., 7th ed. New York ;London: McGrawHill, 2002.

[15] B. Ohler, "Practical Advice on the Determination of Cantilever Spring Constants," 2010. http://nanoscaleworld.bruker-axs.com/nanoscaleworld/media/p/143.aspx.

[16] M. Salerno and I. Bykov, "TUTORIAL: Mapping Adhesion Forces and Calculating Elasticity in Contact-Mode AFM," Microscopy and Analysis SPM supplement, John Wiley and Sons, Itd, 2006.

[17] C. A. Clifford and M. P. Seah, "Quantification issues in the identification of nanoscale regions of homopolymers using modulus measurement via AFM nanoindentation," Applied Surface Science, vol. 252, no. 5, pp. 1915-1933, 2005.

[18] M. A. Monclus, T. J. Young, and D. Di Maio, "AFM indentation method used for elastic modulus characterization of interfaces and thin layers," Journal of Materials Science, vol. 45, no. 12, pp. 3190-3197, 2010.

[19] D. C. Lin, E. K. Dimitriadis, and F. Horkay, "Robust Strategies for Automated AFM Force Curve Analysis-I. Non-adhesive Indentation of Soft, Inhomogeneous Materials," Journal of Biomechanical Engineering, vol. 129, no. 3, p. 430, 2007.

[20] S. Khanna, R. Winter, P. Ranganathan, S. Yedla, M. Kalukanimuttam, and K. Paruchuri, "Sample preparation techniques for nano-mechanical characterization of glass fiber reinforced polyester matrix composites," Composites Part A: Applied Science and Manufacturing, vol. 34, no. 1, pp. 53-65, 2003.

[21] T. J. Young, M. Monclus, W. R. Broughton, S. L. Ogin and P. A. Smith. "Characterisation of Interfaces in Micro- and Nano-Composites", Proceedings of ICCM17, the International Conference on Composite Materials, Edinburgh, Scotland. 2009 


\section{FIGURES}

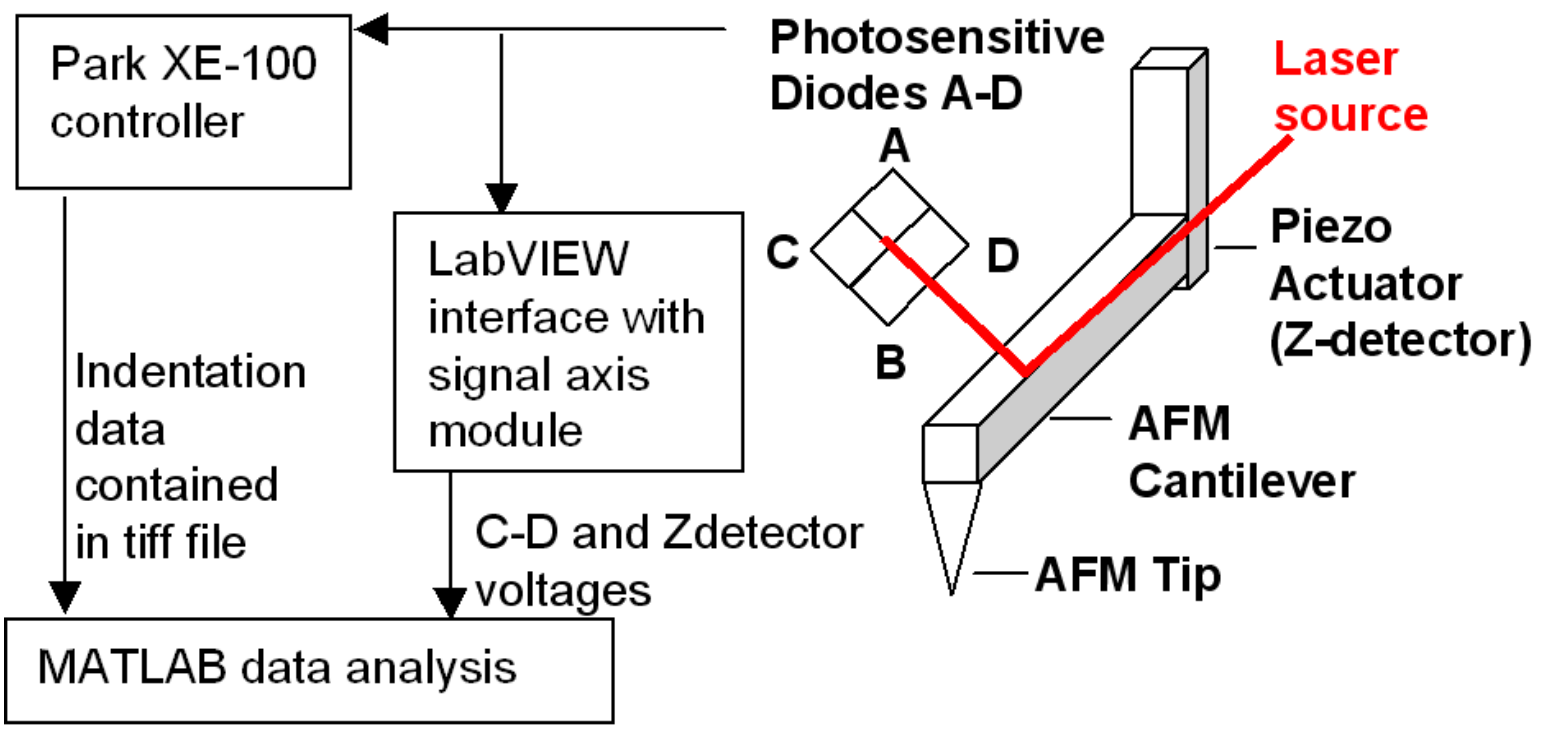

Figure 1: Schematic diagram of the cantilever deflection detector, experimental-setup and analysis

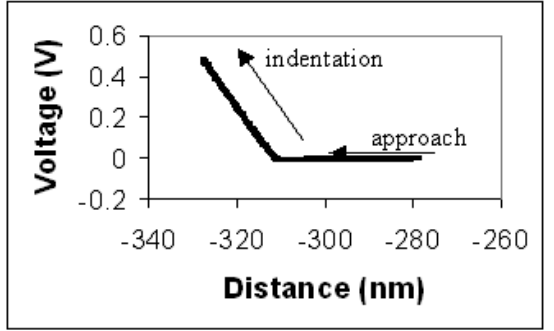

a)

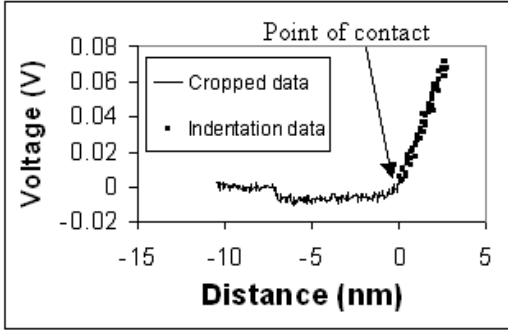

b)

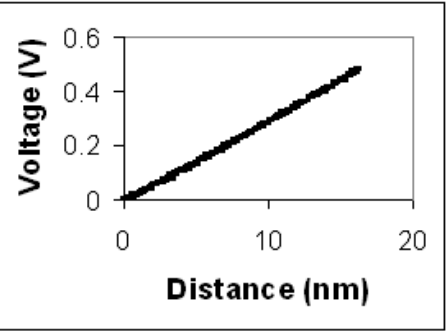

c)

Figure 2: (a) Raw voltage-distance AFM indentation curve from a sapphire surface, (b) the same curve magnified, the sign convention changed to positive and centred about the point of contact and (c) the voltage-distance AFM indentation curve after cropping.

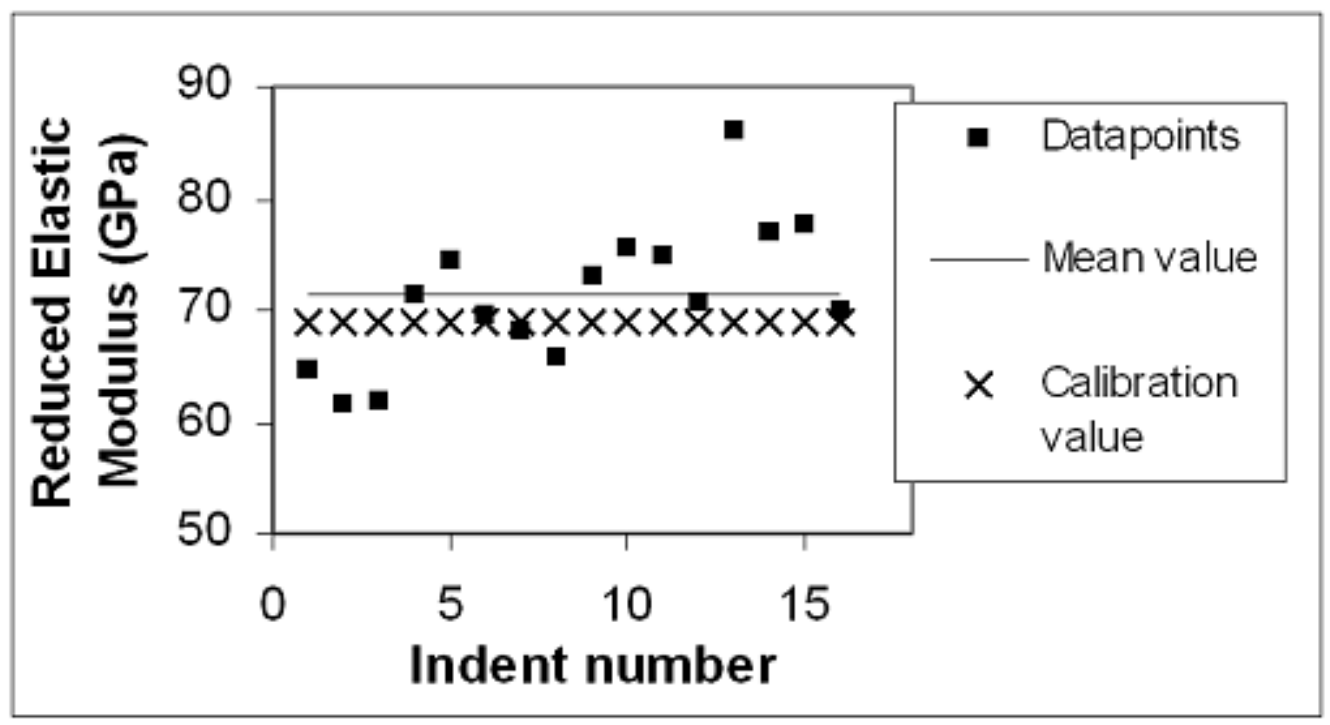


Figure 3: Indentation results on fused silica reference sample after indentation

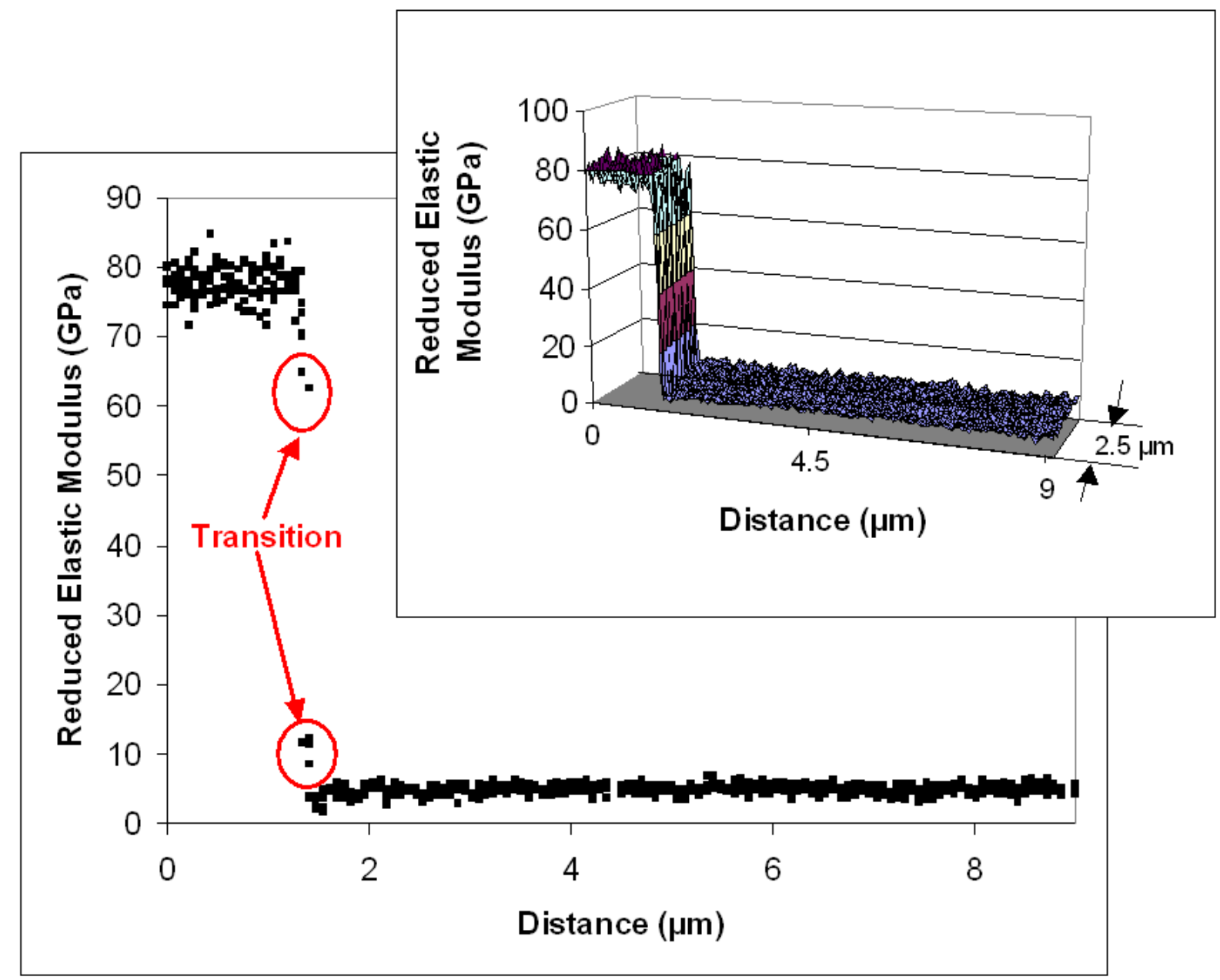

Figure 4: Five lines of indentations over the interface of the glass flake-reinforced polypropylene sample. Transition indentations of intermediate reduced elastic modulus are highlighted. The insert shows the full $128 \times 32$ indentations over an area of $9 \mu \mathrm{m} \times 2.25 \mu \mathrm{m}$. 


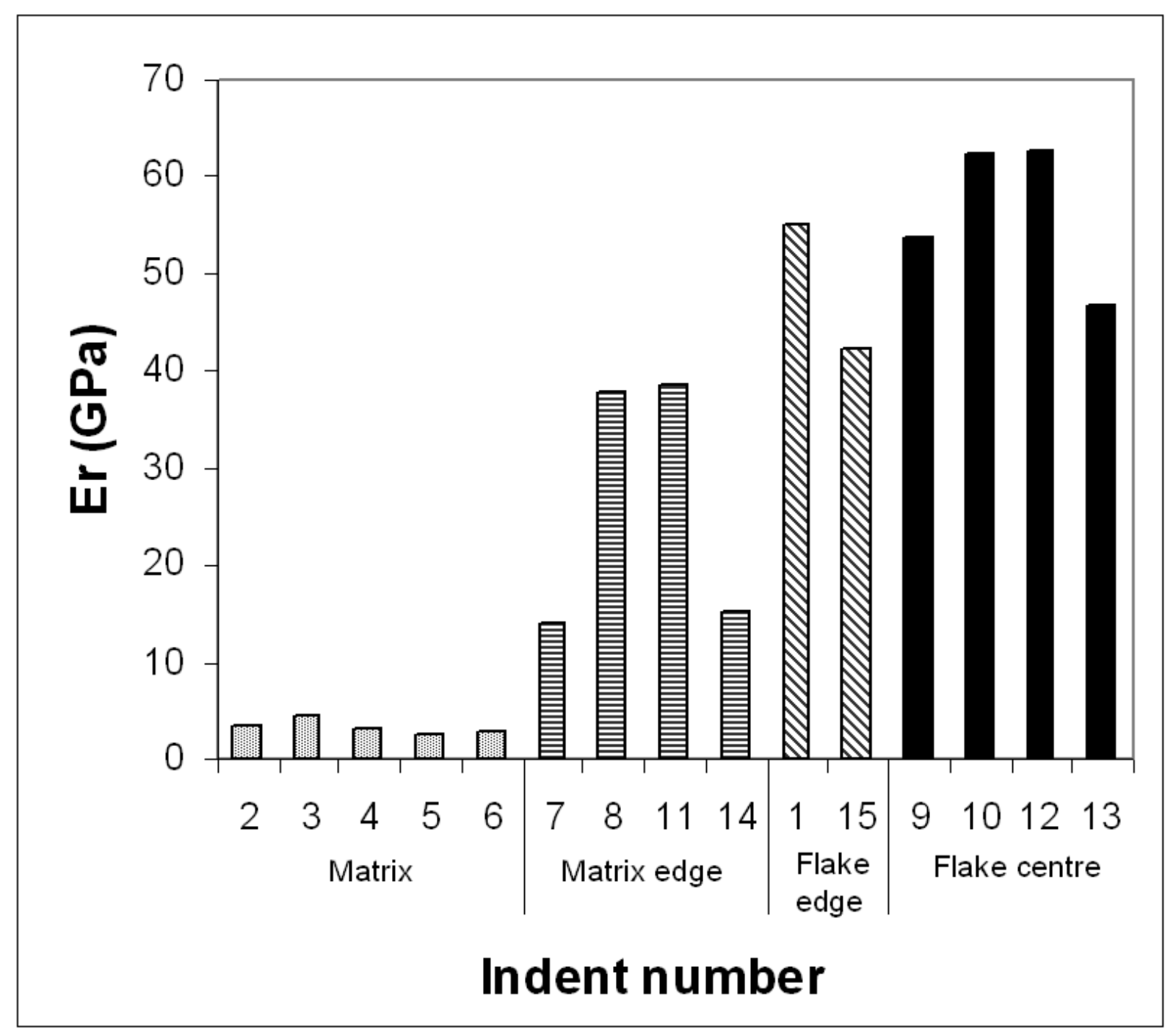




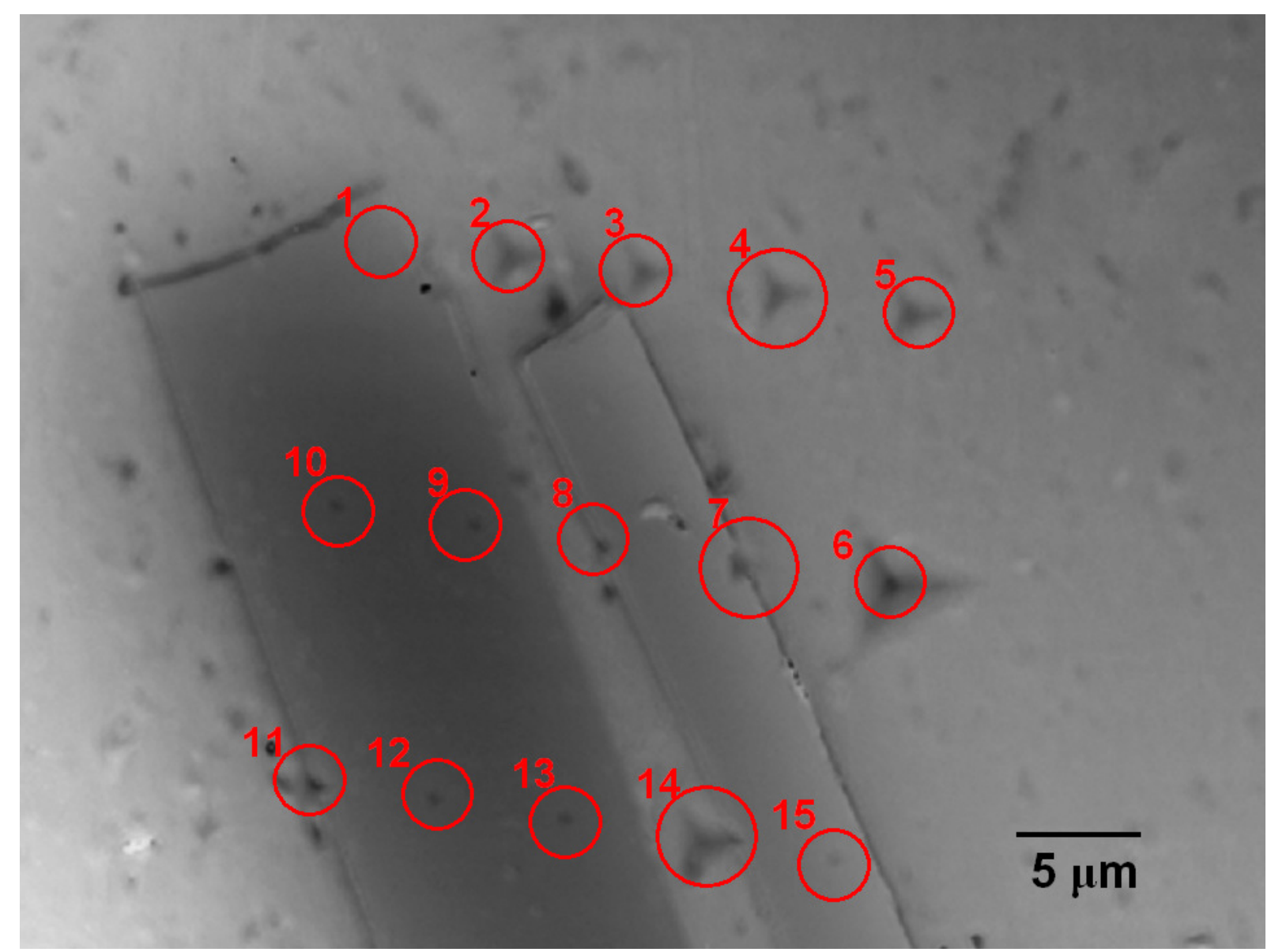

Figure 5: (a) IIT measurements of the glass flake-reinforced polypropylene composite and (b) an optical image of the locations of each residual indentation 


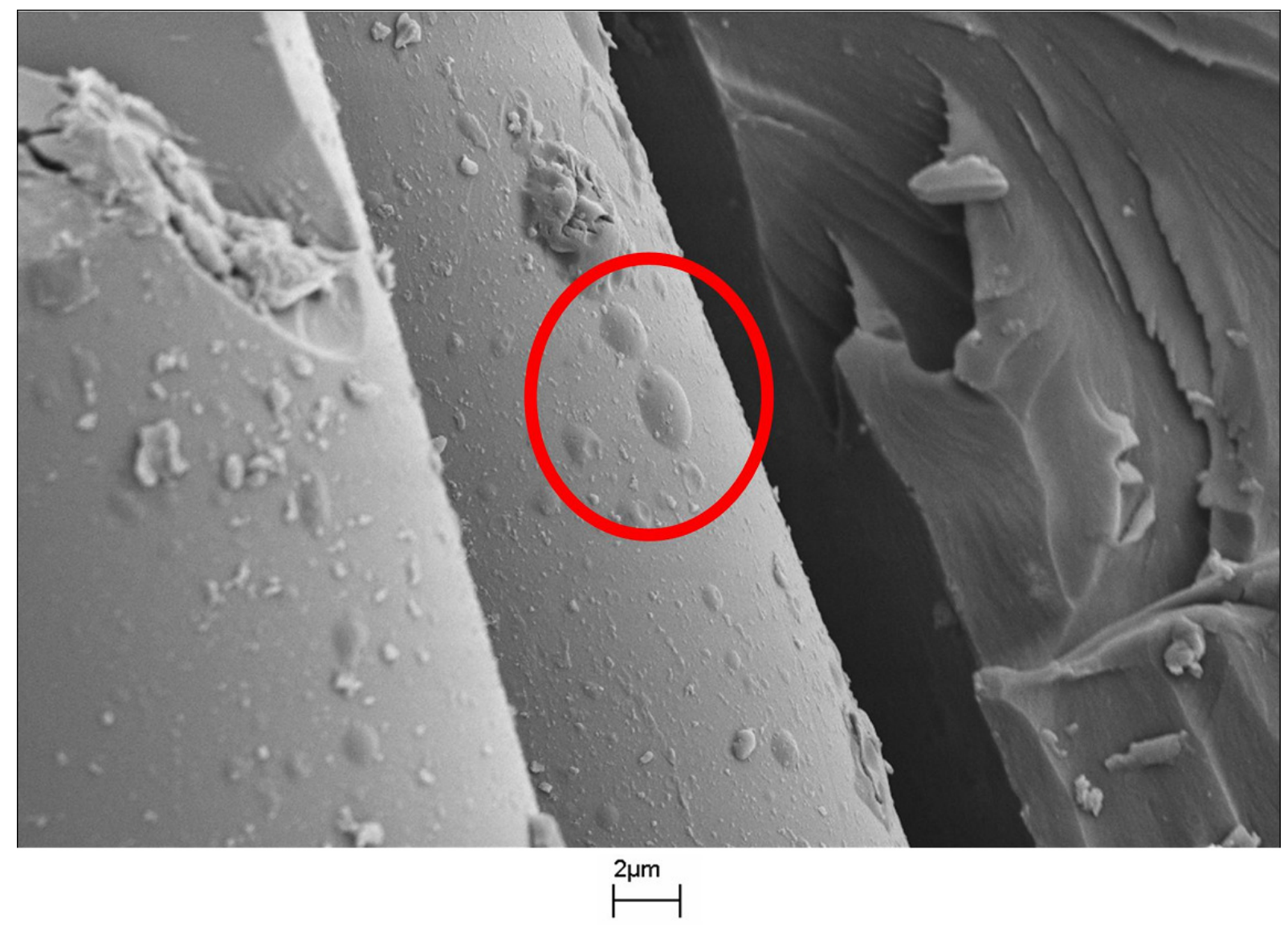

Figure 6: Scanning Electron Microscope image of a poorly bonded glass fibre-reinforced vinylester pultruded rod with coupling agent droplets randomly dispersed over the surface.
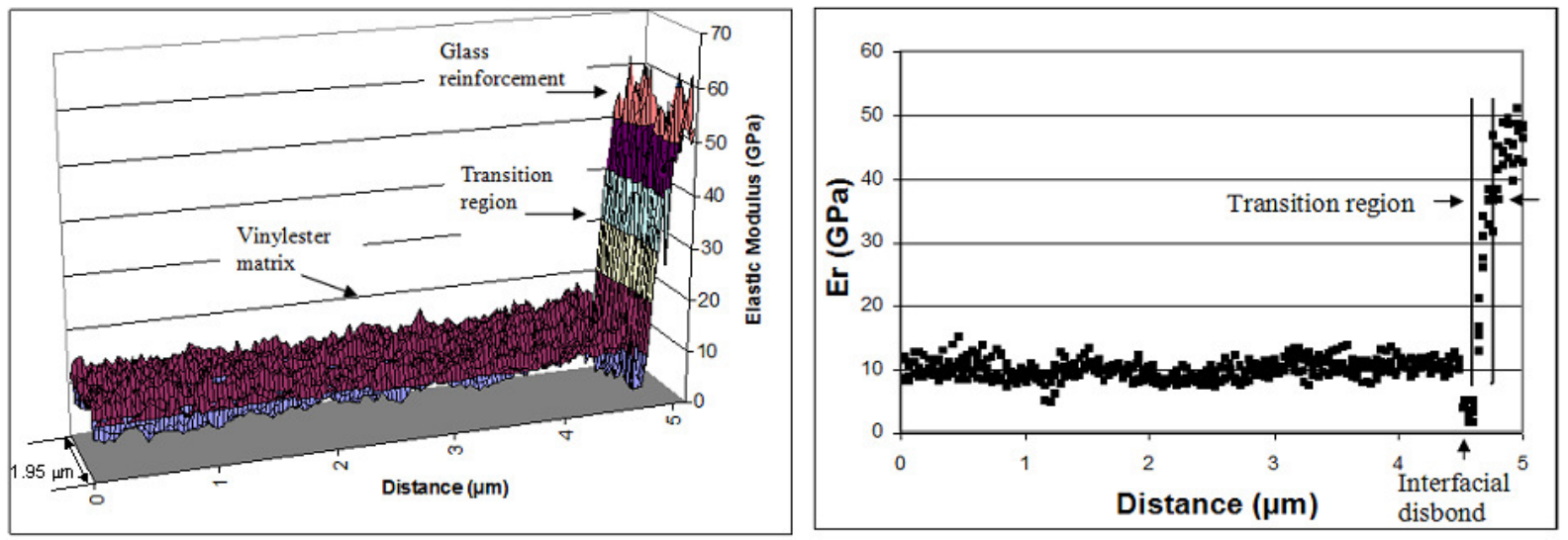

Figure 7: AFM indentation results for the glass fibre-reinforced vinylester with poor interfacial bonding. (a) $96 \times 50$ lines corresponding to an area of $5 \mu \mathrm{m} \times 1.95 \mu \mathrm{m}$ and (b) three lines of AFM indentations taken from (a) illustrating the transition region and interfacial disbond.

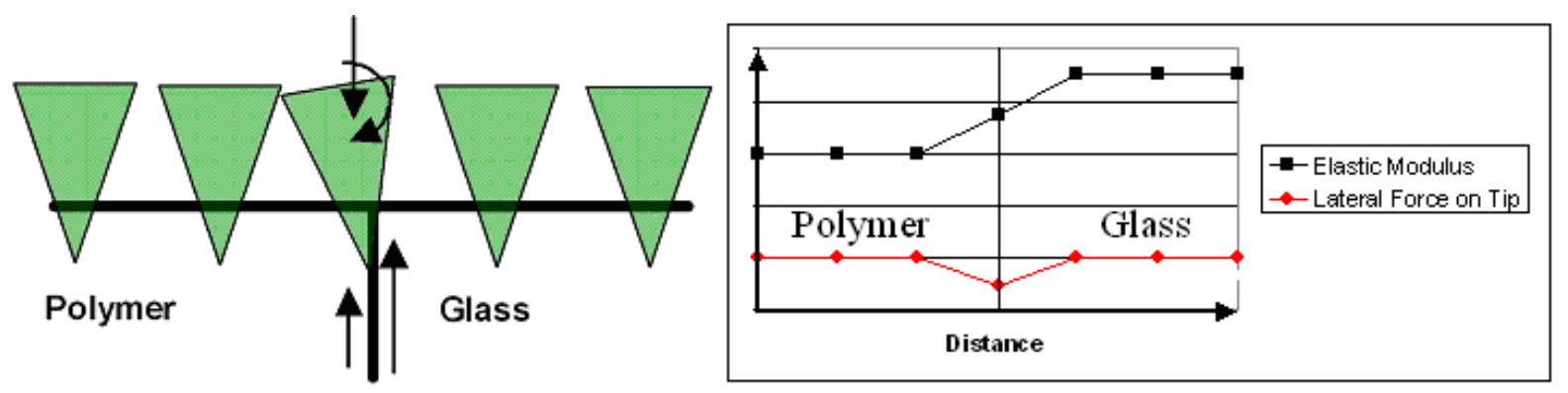


Figure 8: Schematic diagram of uneven loading on the indenter tip resulting in tip twist during indentation and corresponding expected results.

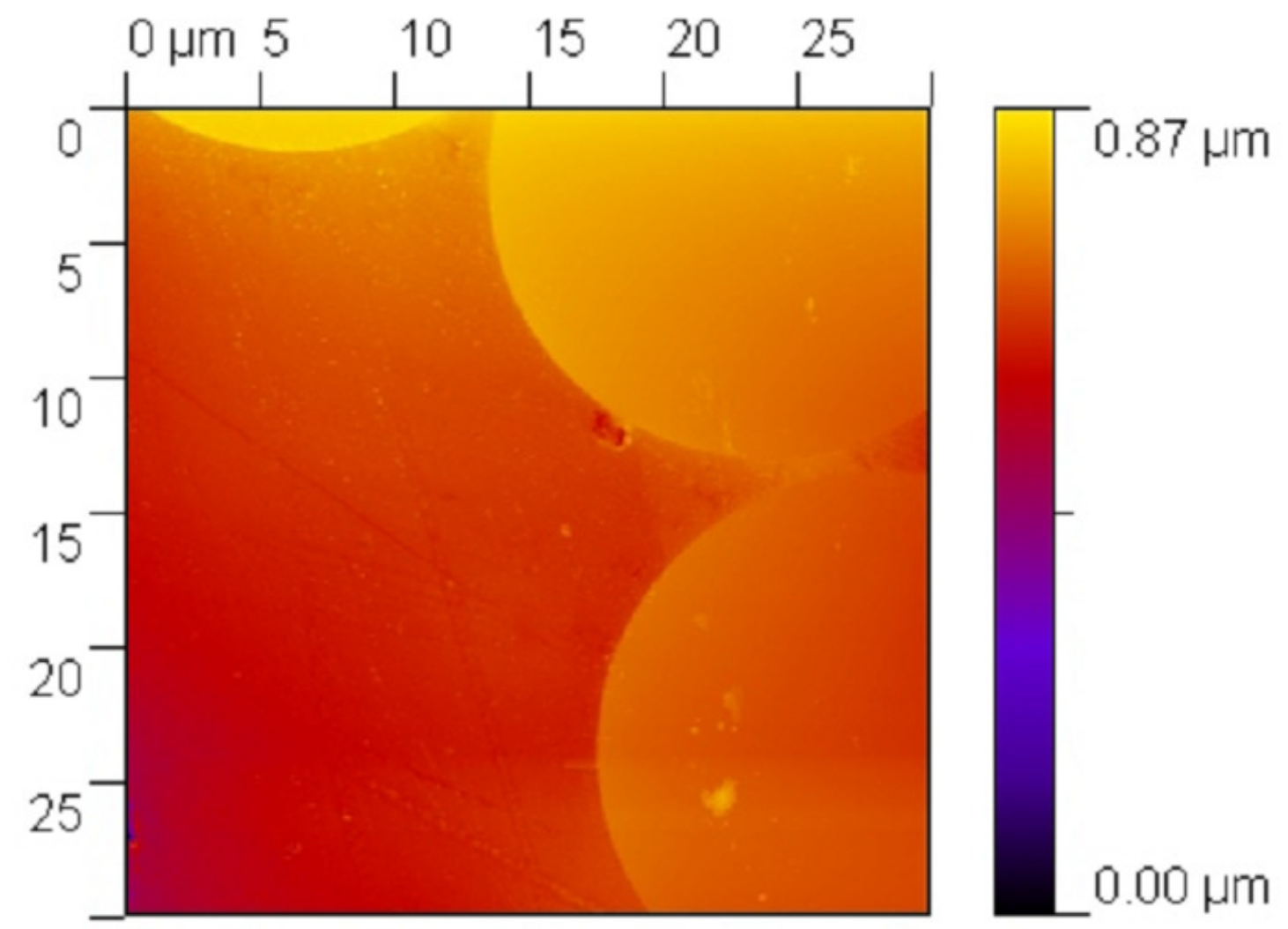

Figure 9: $30 \mu \mathrm{m}$ topographic non-contact AFM image of the glass fibre-reinforced phenolic specimen surface. 


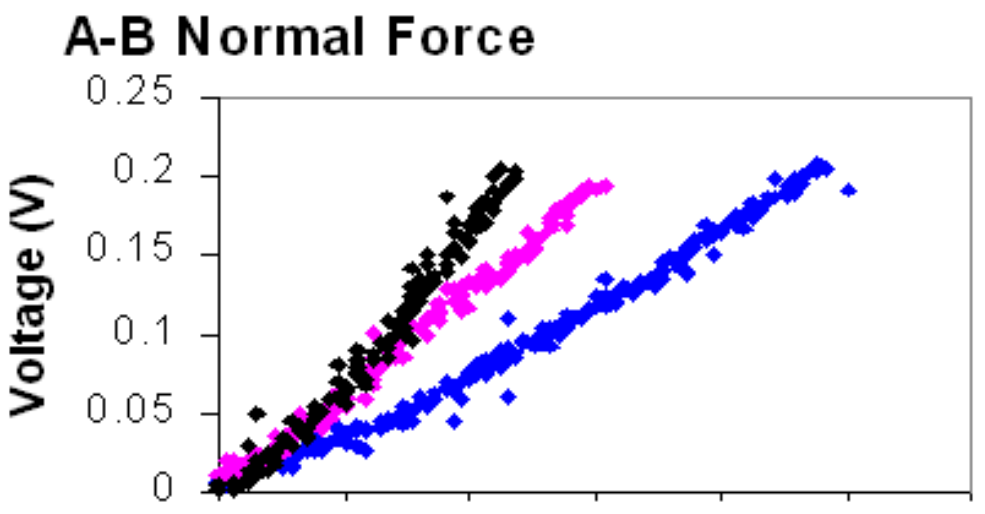

\section{C-D Torsion Force}

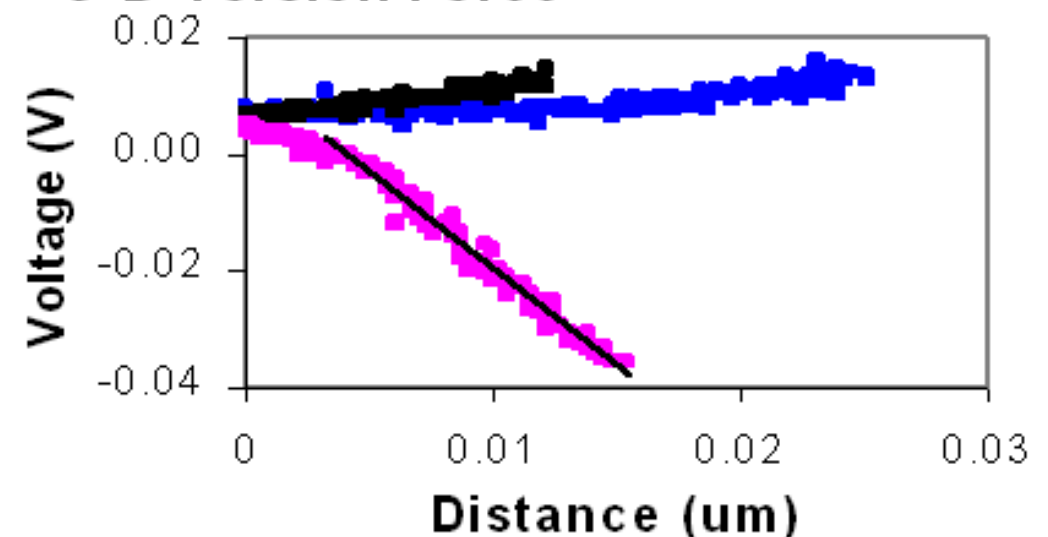

Figure 10: Example raw normal cantilever deflection measurements on the phenolic resin, at the interface and the glass-fibre reinforcement 


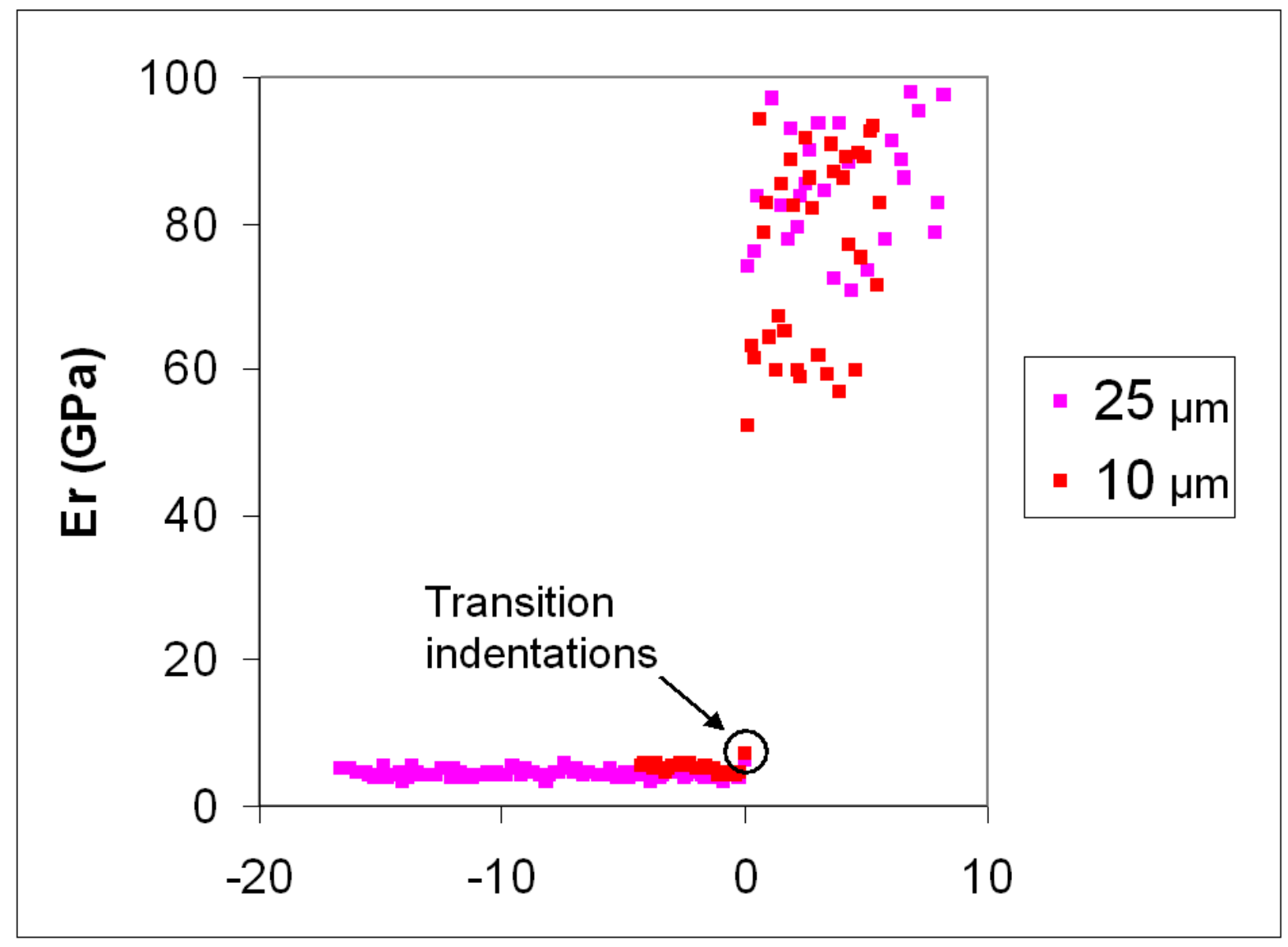

Figure 11: Reduced elastic modulus over the interface of the glass fibre-reinforced phenolic over $25 \mu \mathrm{m}$ (128 indentations) and $10 \mu \mathrm{m}$ (64 indentations) 


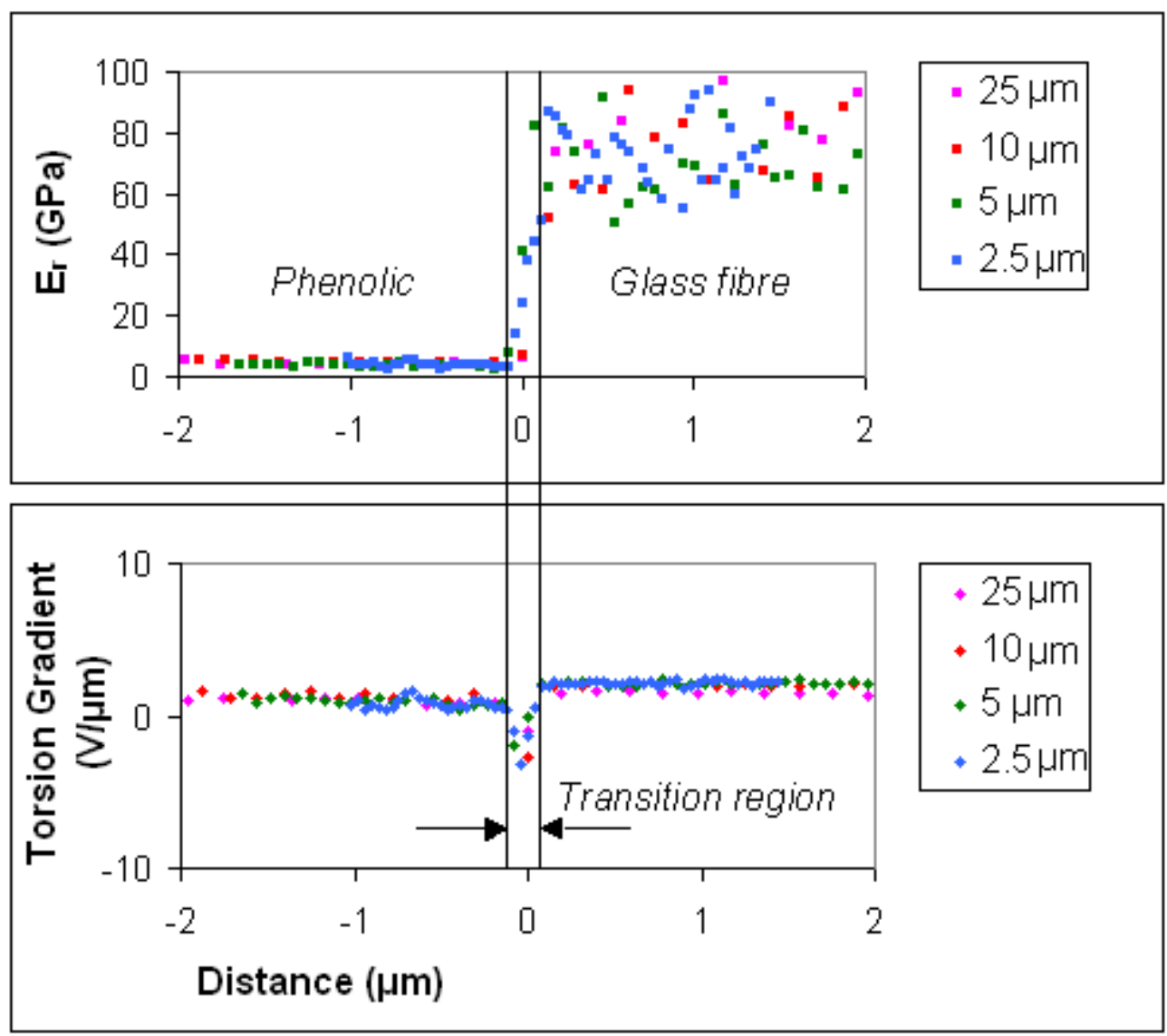

Figure 12: Reduced elastic moduli (GPa) and torsion gradients for each line of indentations over the interface of the glass fibre-reinforced phenolic composite. The results show a 150 $\mathrm{nm}$ transition zone. 


\section{TABLES}

Table 1: SEM measured geometry and calculated mechanical properties of the AFM cantilever and tip (standard deviations of measurements included).

\begin{tabular}{|l|c|}
\hline Parameter & Measured Value \\
\hline Thickness $(\mu \mathrm{m})$ & $16.0 \pm 0.2$ \\
\hline Length $(\mu \mathrm{m})$ & $448 \pm 1$ \\
\hline Width $(\mu \mathrm{m})$ & $49.6 \pm 1.2$ \\
\hline Elastic Modulus $(\mathrm{GPa})$ & 380 \\
\hline Spring Constant $\left(\mathrm{Nm}^{-1}\right)$ & 214 \\
\hline $\begin{array}{l}\text { Radius }(\mathrm{nm}) \text { (experiments on glass flake- } \\
\text { reinforced polypropylene and glass fibre- } \\
\text { reinforced vinylester) }\end{array}$ & 20.5 \\
\hline $\begin{array}{l}\text { Radius }(\mathrm{nm}) \text { (experiments on glass fibre- } \\
\text { reinforced phenolic) }\end{array}$ & $85 \pm 10$ \\
\hline
\end{tabular}

